\title{
Effectiveness of an Intervention for Colic
}

By: Maureen R. Keefe, RN, PhD, FAAN, Marie L. Lobo, RN, PhD, FAAN, Ann Froese-Fretz, RN, MS, CPNP, Anne Marie Kotzer, RN, Ph D, Gail A. Barbosa, RN, ScD, and William N. Dudley, PhD

Keefe MR, Lobo ML, Froese-Fretz A, Kotzer AM, Barbosa GA, Dudley WN. (2006). Effectiveness of an intervention for colic. Clin Pediatr (Phila), 45(2), 123-33.

Made available courtesy of SAGE PUBLICATIONS LTD: http://cpj.sagepub.com

***Note: Figures may be missing from this format of the document

\begin{abstract}
:
Summary: An intervention for infant irritability or colic was evaluated in a randomized clinical trial. A total of 121 full-term irritable infants ( 2 to 6 weeks old) were randomized to routine care or the home-based intervention program. A third group $(n=43)$ of irritable infants were entered into a post-test-only group. Following the 4-week intervention, the treatment group infants cried 1.7 hours less per day than the infants in the control group $(\mathrm{p}=0.02)$. The findings support the emerging view of infant colic as a behavioral pattern that is responsive to environmental modification and structured cue-based care.
\end{abstract}

\section{Article:}

\section{INTRODUCTION}

Unexplained infant crying or colic remains one of the most commonly encountered and least understood problems during infancy. It is unique in that no one cause has been identified for the persistent fussiness and recurrent crying episodes. Although the persistent unexplained crying episodes can occur throughout the day and night, this particular type of irritability has a characteristic diurnal pattern with increased intensity and duration during the evening hours. This type of infant irritability is reported to occur in approximately $15 \%$ to $25 \%$ of all newborns and is equally distributed among ethnic groups as well as both sexes. ${ }^{1-5}$

Persistent infant crying is a major challenge for families under the best of circumstances. It has also been implicated as a major predisposing factor in cases of shaken-baby syndrome ${ }^{6}$ child abuse, and neglect for high-risk families with limited resources and support systems. ${ }^{7-9}$ In a recent study of mothers with colicky infants $70 \%$ revealed explicit aggressive thoughts and fantasies and $26 \%$ admitted thoughts of infanticide during their infants' colic episodes. ${ }^{10}$ The presence of a crying infant has direct effect on parenting patterns and responses as well as family functioning over time. ${ }^{11,1_{2}}$ Without successful management, persistent unexplained crying in the infant can lead to disorganized parental responses, learned helplessness, maternal depression, and insecure parent- infant attachment. ${ }^{13,14}$ In addition to the parenting burden, the costs to the health care delivery system are significant, as these families consume resources in their efforts to find effective management strategies. ${ }^{15}$

\footnotetext{
* Funded by an R01-NR04661 award from the NIH, National Institute for Nursing Research.
} 
Parental distress is often exacerbated by lack of information regarding the cause of the persistent crying. ${ }^{16,17}$ Inconsistent or ineffective approaches to the management of colic only add to the stress in these families. Current treatments are based on proposed etiologies such as gastrointestinal pain due to reflux or gas, food allergies, or anxious new parents. Several studies have tested various dietary regimens and pharmaceutical approaches with mixed results. ${ }^{18-20}$ These theories and approaches, however, do not explain a large portion of the observed infant behavior and have not yielded effective interventions. In practice, approaches to the diagnosis and management of unexplained infant irritability or colic are too often based on hearsay or anecdotes rather than evidence-based scientific rationale. ${ }^{21}$

The purpose of this study was to test an intervention for managing infant irritability that is based on an alternative view of unexplained infant crying and its origin. In 1988, Keefe ${ }^{21}$ proposed a theoretical model that conceptualized infant colic from a developmental psychobiological perspective. Colic, referred to as irritable infant syndrome, is viewed as a delay or disturbance in the infant's sleep-wake cycling or an infant state regulation disorder. Infant state, as popularized by Brazelton, is conceptualized as a level of arousal continuum ranging from deep, quiet sleep to awake and aroused lusty crying. In the proposed model the colicky or irritable infant has a disorganized or undeveloped sleep-wake cycle that underlies the excessive crying and difficulty initiating sleep. ${ }^{21}$ The process underlying the observed behavioral manifestations is seen as a dysfunction in the infants' ability to self-regulate their state or sleep-wake cycles based on central nervous system immaturity rather than gastrointestinal system dysfunction. This behavior instability may be exacerbated by parental inconsistency and environmental disruptions. From this framework, irritable infants are viewed as sensitive and more easily overstimulated by busy chaotic environments. As they become overwhelmed and fatigued, they cannot self-soothe or reduce their arousal level sufficiently to fall asleep. Parents, while very concerned, may actually be reinforcing the irritable behavior pattern by inconsistent attempts at strategies that are not contingent with the infant's unclear signals and erratic cues. Infant irritability, when viewed as a developmental state regulation disorder that disrupts the synchrony of the parent-infant dyad, necessitates an approach that provides support for the parents and modification of the infant's care and environmental routines during this early sensitive period. ${ }^{21}$ Recent studies exploring behavioral approaches to the management of colic have limited success with compliance and inconsistent results. $^{22-24}$

\section{Program Description}

An individualized intervention program, referred to as the "REST Routine for Infant Irritability" (REST Routine), was developed based on this developmental, biological perspective of colic or infant irritability. The program elements evolved out of previous research by this investigative team over a period of 15 years. ${ }^{21,25-27}$ The primary goal of the newly designed intervention program is to regulate and reduce the infant's level of arousal by environmental and behavioral restructuring. The four specific objectives of this new integrative approach to the management of infant irritability are to: (1) promote synchrony in the parent- infant dyad; (2) decrease intensity and duration of infant irritability; (3) promote state regulation and organization in the infant; and (4) provide information and support to the parents.

The program has two components; the first component consists of activities directed toward the infant, and the second component is for the parents. The four principles guiding the REST 
routine for infants are: Regulation, Entrainment, Structure and Touch. The following is a brief explanation of these four concepts, which the intervention nurse uses to form specific recommendations and care plans for the infant at each of the home visits.

\footnotetext{
Regulation refers to the support required to assist these infants in regulating their state behavior and to protect them frombecoming overstimulated and exhausted during the first few months of life. Entrainment is the process whereby the infant's basic sleep-wake cycles are synchronized with relevant aspects of the environment. Structure and repetition are also key concepts of the REST program. The goal is to create a predictable and recurrent pattern of events for these infants who are not intrinsically well-organized. Touch covers various infant holds and positions that areincorporated into the program.
}

The four concepts of the REST Routine that guide the intervention nurse in working with the parents are: Reassurance, Empathy, Support, and Time-out. These concepts are individualized and applied in some of the followings ways: Reassurance is focused in two areas; the infant's health and the parent's competence. Empathy is provided through listening and acknowledging the challenge of parenting these high-need and high-maintenance infants. Support involves serving as an advocate and resource for parents in obtaining assistance and creating a support network. Time-out legitimizes the primary caregiver's critical need to take care of themselves. A specific period of time-out from parenting (at least 1 hour) is scheduled into each day.

To systematically test this approach, these concepts were operationalized and developed into a month-long, home-based intervention program, the REST Routine. The concepts outlined were applied and individualized by the nurse working in partnership with the needs and unique features of each family. The intervention program consisted of four home visits, provided by master's-prepared pediatric nurse specialists who had been trained in the intervention model. Infant behavior assessments and demonstrations, parent education materials, a video entitled "Fussy Babies and Frantic Families," parent workbook with observation guides and accompanying worksheets, pattern recognition guides, individualized daily schedules, and 5 Fuss Fixer magnets were all developed and included in the REST Routine program for families with irritable infants.

\section{METHOD}

Design

This NIH -funded intervention study employed a two-group, randomized clinical trial experimental design. Data were collect at two sites by using computer-generated randomization lists for the participants at each site. Group assignment for each ID number was generated a priori and controlled centrally to maintain group comparability within site. Data collection team members were unaware of group assignment and participants were informed of their specific protocol plan after informed consent was obtained. The primary research hypothesis tested in this randomized clinical trial proposed that families who received the REST Routine would report less infant irritability and unexplained crying than families who received routine well-child care. Infants were randomly assigned to the treatment or control group and repeated outcome assessments were made over the 8- week study period.

\section{Subjects}

Data collection sites were Charleston, South Carolina, and Denver, Colorado. The families that comprised the study sample were referred by their pediatrician, nurse referrals, or recruited 
through local advertisement and flyers regarding the program. One hundred twenty-one infants and parents were recruited based on the following criteria: full term, healthy low-risk infants between the ages of 2 and 6 weeks, and living within a 2-hour radius of the metropolitan area. In addition the infants were prescreened for amount of unexplained crying by using a brief phone intake interview. The study enrollment requirement was set at an average of 3 hours per day or more of unexplained crying over the past week or two as a minimum. Infants meeting all the above criteria were enrolled and randomly assigned to receive either the REST Routine or standard well-child care for a 4-week period. A separate trained evaluation team member visited the home to obtain measurements upon study entry, immediately following the 4- week intervention, and at the 8- week follow-up visit. Of the 180 infant/parent participants, 16 withdrew from the study or were lost to follow-up due to relocation or return to work. Those who withdrew were no different in infant gender, gestational age, maternal and parental ages, and were equally distributed across states and treatment and control conditions. The data from these participants were excluded from the analyses.

\section{Post-Test-Only}

A third group of infants was added to the study as parents in the control group began to comment on the benefits derived from having a nurse visit their home. This post-test-only group consisted of infants referred to the study who met the criteria but were older than the 2- to 6-week intake age requirement. Their age was comparable to those infants completing the intervention program. These infants were included in a subsequent analysis and serve to estimate or explore change in the control group due to attention or developmental changes alone.

The post-test-only group, which received only one home visit to obtain outcome data, was comprised of 43 infants. The mean age of the infants in the post-test-only group was $10.9 \pm 2.1$ weeks. Demographics for all groups of participants are provided in Table 1.

All infants enrolled in the study were full-term (gestational age $=39 \pm 1.4$ weeks) infants who had been screened for illness and organic causes for their unexplained excess irritability. The mean birth weight of the infants in the study was $7.3( \pm 0.9)$ pounds. Gender distribution was 


\section{SUBJECT DEMOGRAPHICS}

\begin{tabular}{lccc} 
& Treatment $(\mathrm{n}=64)$ & Control $(\mathrm{n}=57)$ & Post-Test-Only $(\mathrm{n}=43)$ \\
\hline State of residence & & & \\
- South Carolina & $23(35.9 \%)$ & $17(29.8 \%)$ & $6(14.0 \%)$ \\
- Colorado & $41(64.1 \%)$ & $40(70.2 \%)$ & $37(86.0 \%)$ \\
Gender & & & \\
- Male & $30(46.9 \%)$ & $25(43.9 \%)$ & $27(62.8 \%)$ \\
- Female & $34(53.1 \%)$ & $32(56.1 \%)$ & $16(37.2 \%)$ \\
Age at entry in weeks & $5.06( \pm 1.31)$ & $5.10( \pm 1.30)$ & $10.9 \pm 2.1$ \\
Ethnicity & & & \\
- Asian or Pacific Islander & $6.3 \%$ & $3.5 \%$ & $0.0 \%$ \\
- Black & $6.3 \%$ & $7.0 \%$ & $2.8 \%$ \\
- Hispanic & $4.8 \%$ & $17.5 \%$ & $5.6 \%$ \\
- White & $81.0 \%$ & $70.2 \%$ & $91.7 \%$ \\
- Other & $1.6 \%$ & $1.8 \%$ & $0.0 \%$ \\
Feeding method & & & \\
- Breast & $45.3 \%$ & $46.4 \%$ & $32.6 \%$ \\
- Bottle & $18.8 \%$ & $21.4 \%$ & $20.9 \%$ \\
- Breast and bottle & $35.9 \%$ & $30.4 \%$ & $46.5 \%$ \\
Birth weight in pounds & $7.5(1.02)$ & $7.3(.86)$ & $6.9(0.67)$ \\
Gestational age in weeks & $39.4(1.27)$ & $38.9(1.46)$ & $38.5(1.35)$ \\
Mother's age & $31.2(6.16)$ & $31.1(4.55)$ & $30.7(4.98)$ \\
Parity & & & \\
- Primiparous & $48.4 \%$ & $47.4 \%$ & $48.8 \%$ \\
- Multiparous & $51.6 \%$ & $52.6 \%$ & $51.2 \%$ \\
Mother's education in years & $14.9(2.27)$ & $15.6(2.39)$ & $15.3(2.68)$
\end{tabular}

equal with 82 female infants and 82 male infants. The treatment group consisted of 64 infants and the control group had 57 infants. The age of the infants at baseline data collection ranged from 2.6 weeks to 7.7 weeks of age. The mean age at time of enrollment was $5.1 \pm 1.3$ weeks. The average age of the parents in the study was $31.0( \pm 5.3)$ years for mothers and $33.2( \pm 6.7)$ years for fathers. The families were predominantly well- educated, middle class, and white. There were no statistically significant group differences found in any of the descriptive demographic variables or any of the crying variables at baseline.

\section{Measures}

\section{Fussiness Rating Scale}

For the purpose of this investigation, infant irritability was defined as a behavior disorder that is characterized by recurrent episodes of unexplained fussiness and crying that is associated with restlessness and diminished soothability. Unexplained infant irritability was measured by using the Fussiness Rating Scale (FRS) that has been employed in previous research. ${ }^{25-27}$ This tool was 
completed by the parent at the initial visit to obtain baseline data. Weekly parental ratings were obtained during the 4-week intervention phase and continued throughout the 4-week follow-up phase.

The FRS is a parent report scale that is designed to measure unexplained early infant crying or colicky behavior. This tool is a modification of the intensity rating scale originally developed by Emde and Gaensbauer. In previous work it was found to be easier to complete and interpret than the parent diary forms that employ the use of symbols. The scale stem first defines fussiness as a state of irritability that is not explained by a cause such as hunger or pain from a shot. The form also states that fussiness may include crying, fussing, and restlessness. The parent is then asked to rate their baby's typical fussy behavior over the past day or week. The following three aspects or dimensions of infant fussiness are individually rated: (1) hours of unexplained crying per day, (2) intensity of fussiness, and (3) amount of fussiness per day. ${ }^{27}$ For the hours of unexplained crying, parents were asked to estimate the average number of hours per day over the previous week that their infant cried. Parents indicated the typical intensity of fussiness and amount of fussiness per day by circling a number on a 0 to 6 visual analog scale $(0=$ no fussiness and $6=$ constant fussiness). An additional open-ended section for comments was also provided on the rating sheet. In this study parents filled out the FRS form once a week for 8 weeks following the baseline data entry visit. The FRS was originally adapted from the Intensity Rating Scale developed by Emde and associates. ${ }^{28}$ The scale has been used in a previous predictive study as the outcome criteria and in a pilot study as an outcome measure for the intervention being tested. Inter-rater reliability among the items when scored by both parents independently during the predictive study ranged from 0.71 to $0.86 .^{28}$ For the pilot study an item was added that asked the parent the number of fussy episodes the infant had per week. Response options ranged from no episodes this past week to every day. This item did not add consistent or meaningful data and was deleted in this study. Independent parent ratings of infant fussiness revealed an inter- rater correlations ranging from $r=0.72$ to 0.91 in the pilot study.

\section{Resolution of Irritability}

The impact of the treatment on level of infant irritability was also evaluated by measuring of resolution of unexplained, excessive crying. During the exit interview conducted at the 8-week follow-up visit, parents were ask if their infant's unexplained crying episodes had diminished or resolved. Response options were: "Not Resolved," Partially Resolved," and "Completely Resolved." If irritability was resolved, parents were asked to indicate the infant's age at the time of improvement or resolution.

\section{Statistical Analysis}

Data were entered from the data collection forms by using the data entry function of EpiInfo (Centers for Disease Control, Atlanta, GA). After all data had been entered, the data were reentered to identify any data-entry errors. Any discrepancies from the first and second data entry were indicated by the program and corrected before proceeding with the analysis. All data were analyzed by using SAS (version 8.02; SAS Institute, Cary, NC). Examination of the distributions indicated that the outcome measures were not normally distributed and transformations did not completely correct the non-normality in the data set. Thus, a conservative approach was taken to the analyses by using nonparametric alternatives to tests of group differences. Measures taken over time were analyzed by using Generalized Estimating 
Equations ${ }^{29}$ with time of measurement as a repeated within-subjects variable and hours crying at intake used as a covariate. Hours of unexplained crying reported at phone intake was included as a covariate in the repeated-measures analysis to reduce unaccounted for variance. The analyses were conducted with SAS proc GENMOD ${ }^{29}$ Measures taken at only one time point, the exit interview, were also analyzed with proc GEN- MOD with a cumulative logistic approach. ${ }^{30}$ This analytic strategy was used to account for the developmental trajectory as we examined the treatment effects.

\section{RESULTS}

Hours of Crying

Initial unexplained infant crying in both treatment and control groups was reported to be almost 6 hours per day ( $5.9 \pm 3.5$ hours). The average number of hours crying at baseline for the treatment group was 5.5 hours, ranging from 2.5 to 12 hours. The average number of hours of crying at baseline for the control group was 5.9 hours, ranging from 2.5 hours to 20 hours. The difference in average hours of crying between the two groups at baseline was not statistically significant. The average hours of crying per day for 5 infants included in the study ( 3 treatment group and 2 control) dropped below the 2.5- hour eligibility criteria between the intake phone call and initial baseline assessment. Data from these infants were retained in the final analysis as removal did not affect the results.

The Generalized Estimating Equations ${ }^{29}$ analysis of the intervention effect on amount of crying over 9 weeks was conducted with the square-root transform of hours crying. The analyses showed a main effect of Time, $\mathrm{x}^{2}(1)=75.61, \mathrm{p}<0.001$; no main effect of Treatment, $\mathrm{x}^{2}(1)<1$, n.s.; but a Treatment by Time interaction, $\mathrm{x}^{2}(1)=5.53, \mathrm{p}=0.02$. Post hoc testing using polynomial contrasts showed the locus of this interaction to be at the 4-week follow-up visit ( $\mathrm{p}=$ $0.04)$.

Initial infant crying levels in both treatment groups were reported to be over 5 hours per day of unexplained crying $(5.7 \pm 3.2)$. Both groups showed a decrease in crying over the 8-week study period. However, infants in the REST Routine treatment group cried 1.7 hours less per day at study completion than the control group infants $(\mathrm{p}=0.02)$. At the 8-week follow-up visit the mean for the treatment group was $1.29 \pm 1.21$ and the control mean was $2.94 \pm 3.17(\mathrm{p}<0.02)$. The post-test-only group reported average daily crying times of $5.9 \pm 4.3$ hours. This was significantly higher than both the treatment and routine care group at that time period $(p<0.001)$ (Figure 1).

\section{Intensity of Fussiness}

At baseline the mean ratings of intensity of fussiness on the 7- point Lickert scale were $4.67 \pm$ 0.94 for the treatment group and $4.51 \pm 1.07$ for the control group, essentially no difference. The analysis of the intervention effect on intensity of fussiness showed a main effect of Time, $x^{2}(1)=$ 69.26, $\mathrm{p}<0.001$; no Treatment effect, $\mathrm{x}^{2}(1)<1$, n.s.; and a significant Treatment by Time interaction, $\mathrm{x}^{2}(1)=4.37, \mathrm{p}=0.04$. The average intensity score for the treatment group on the 0 to 6 response scale at the 8 -week follow-up visit was $1.97 \pm 1.32$ and it was $2.79 \pm 1.83$ for the control group

(Figure 2). 


\section{Resolution of Irritability}

For purposes of this analysis resolution of infant irritability was set at a weekly average of 1 hour or less per day of unexplained crying or fussiness. Using this criterion the analysis of the intervention effect on resolution of crying

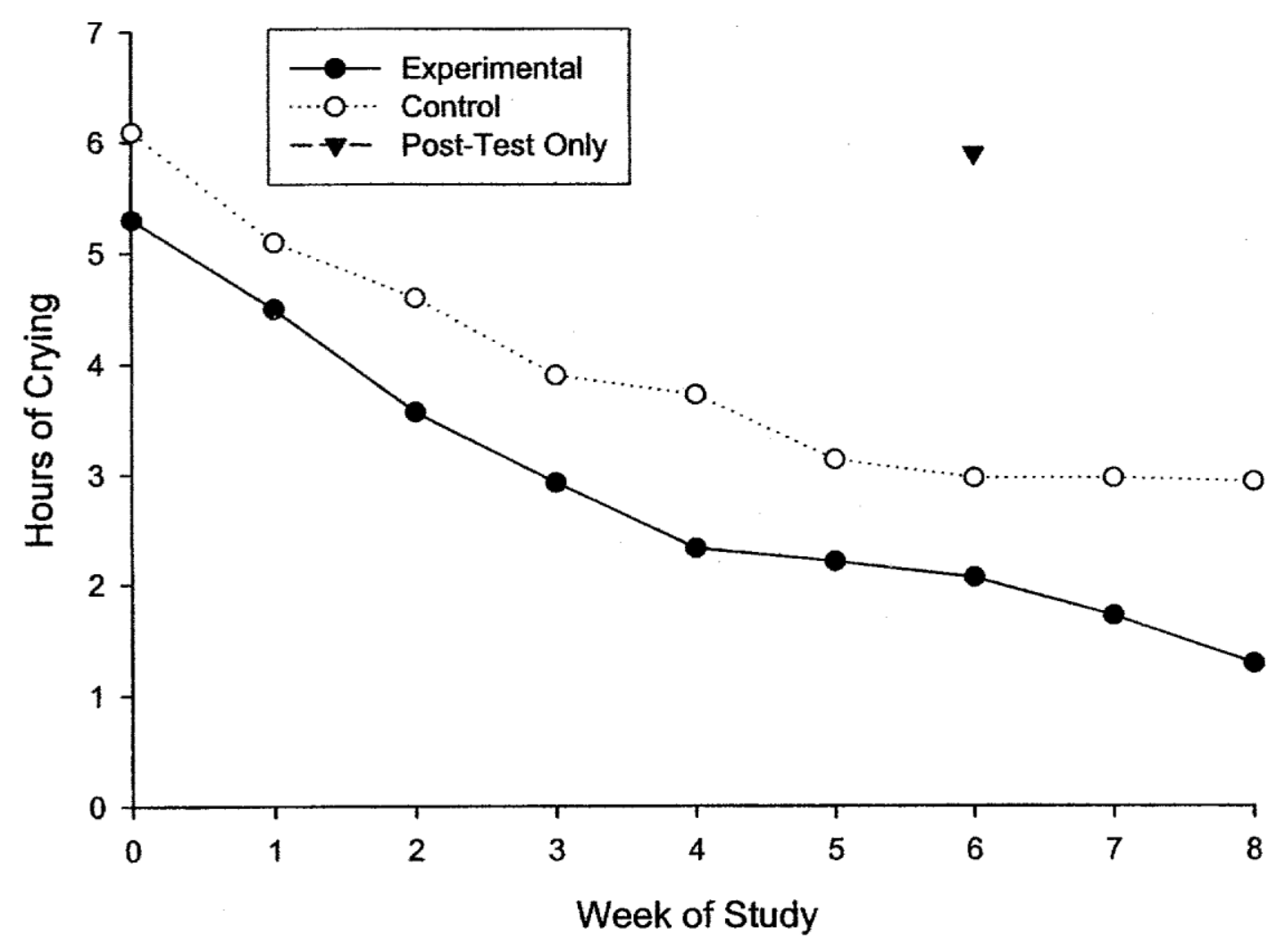

Figure 1. Comparison of group means for average daily hours of crying. 


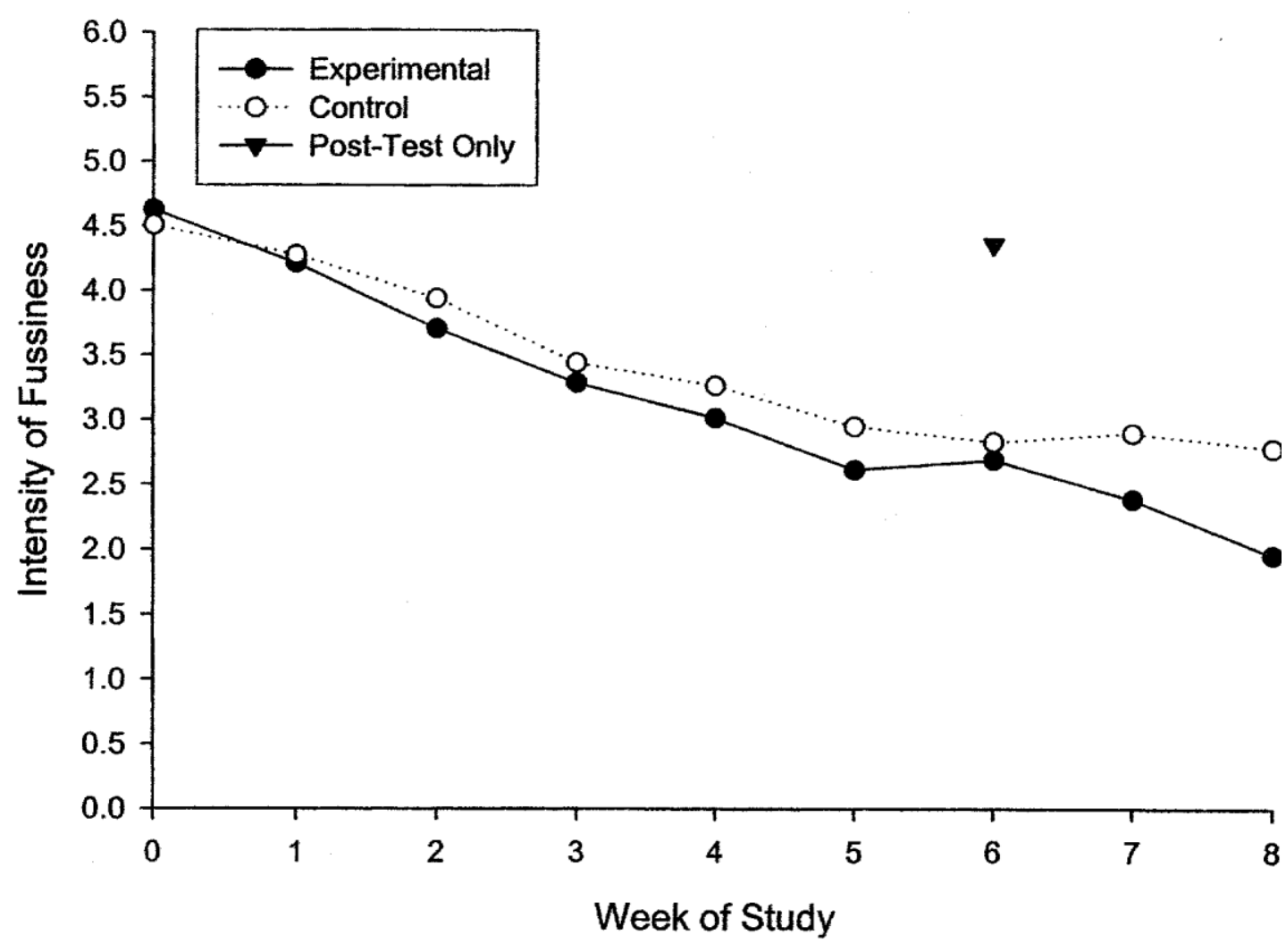

Figure 2. Comparison of group means for intensity of fussiness ratings.

as computed from hours of crying and converted to a percentage resolved for both the treatment and the control groups. The analysis revealed a main effect of Time, $\mathrm{x}^{2}(1)=15.92, \mathrm{p}<0.001$; no Treatment effect, $\mathrm{x}^{2}(1)<1$, n.s.; and a Treatment by Time interaction, $\mathrm{x}^{2}(1)=4.85, \mathrm{p}=0.03$. As can be seen in the graph, $61.8 \%$ of the treatment group infants were resolved and only $28.8 \%$ of the control group were resolved at the 8-week follow-up visit using this operational definition (Figure 3).

Analysis of the 8-week outcome data for all three groups with age as a covariate showed a significant main effect of Treatment, $\mathrm{x}^{2}(2)=46.80, \mathrm{p}<0.001$. Planned contrasts showed that the post-test-only group was significantly higher than the treatment group, $\mathrm{x}^{2}(1)=43.49, \mathrm{p}<0.001$; that the post-test-only group was significantly higher than the control group, $\mathrm{x}^{2}(1)=22.44, \mathrm{p}<$ 0.001 ; and that the control group was significantly higher than the treatment group, $\mathrm{x}^{2}(1)=13.87$, $\mathrm{p}<0.001$. The observed means for untransformed hours of crying are shown in Table 2 . Analysis of the intensity ratings at 8 weeks for all three groups with age as covariate showed a significant main effect of Treatment, $\mathrm{x}^{2}(2)=16.10, \mathrm{p}<0.001$. Planned contrasts showed that the post-test-only group was significantly higher than the treatment group, $\mathrm{x}^{2}(1)=43.49, \mathrm{p}<0.001$; that the post-test- only group was significantly higher than the control group, $\mathrm{x}^{2}(1)=22.44, \mathrm{p}<$ 
0.001 ; and that the control group was significantly higher than the treatment group, $\mathrm{x}^{2}(1)=13.87$, $\mathrm{p}<0.001$. The observed means for Intensity of the Fussiness are shown in Table 2.

\section{Program Evaluation}

Both qualitative comments and ratings of the participants receiving the individualized, homebased intervention program were very positive. Parents reported they were able to develop strategies that helped prevent their infants' fussy episodes as well as minimize the severity. Overall the

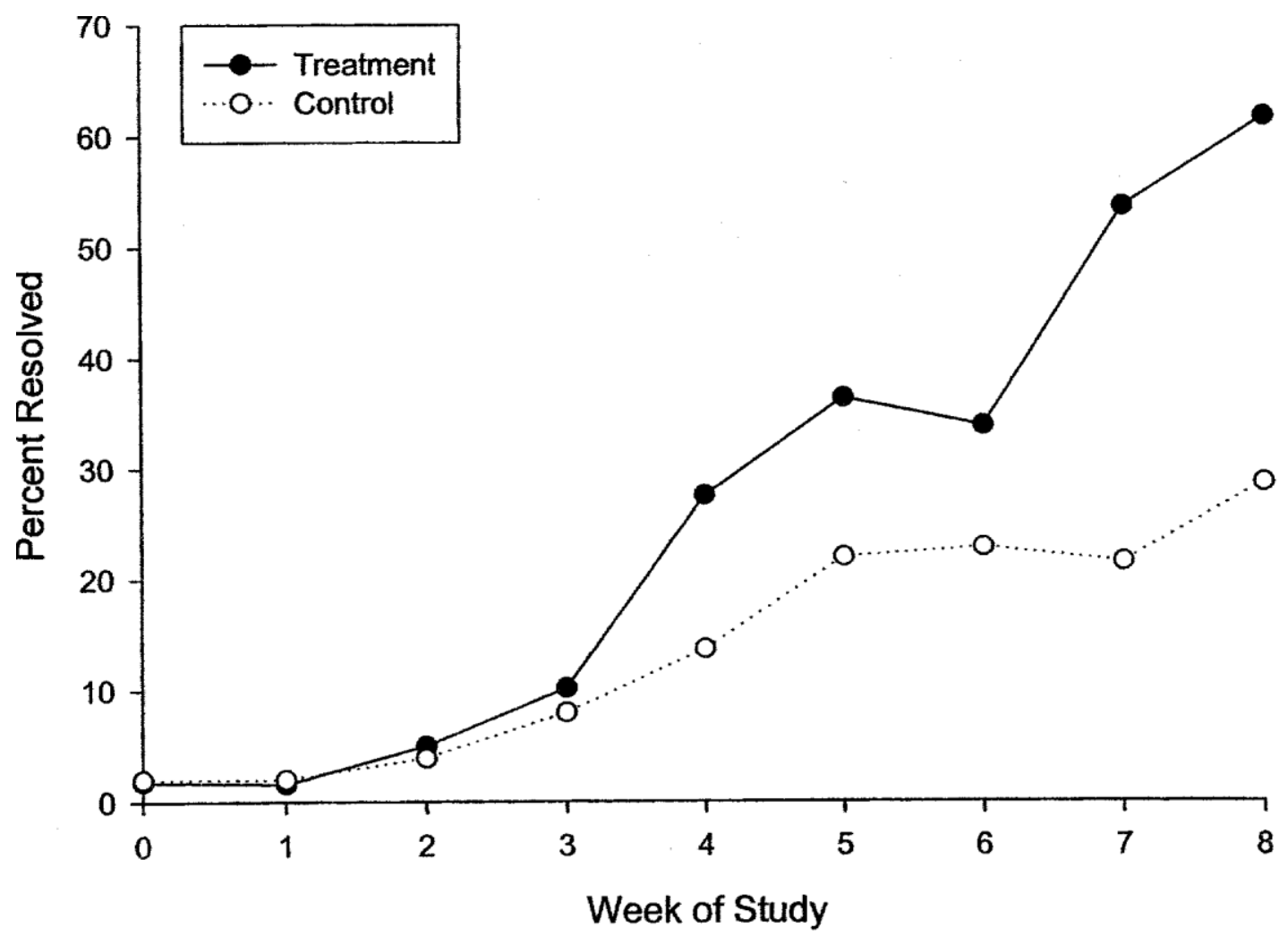

Figure 3. Computed resolution of crying by group.

parents evaluated the intervention as very helpful. Particular components of the program that were noted as most helpful focused on the home visits by a nurse $(88.5 \%)$, and other information provided. Parents particularly valued having someone to listen and serve as a resource for their questions (84\%). Sharing experiences of other parents with irritable infants $(77.4 \%)$ and calling to check on them between visits were also perceived as very helpful (67.7\%). Information regarding daily routines, infant cues, and techniques to help regulate their infant's states was identified as very useful new information for these parents. Families indicated they would be interested in establishing some type of phone network (75.5\%) or support group for parents of irritable infants (72.7\%). Other recommended additions included weight checks and parenting classes. 


\section{Exit Interviews}

Exit interviews were conducted on all subjects at the conclusion of the 8-week study period. Responses to the exit interview items regarding overall satisfaction with care showed a sig-

\begin{tabular}{|c|c|c|c|}
\hline \multicolumn{4}{|c|}{ COMPARISONS WITH POST-TEST-ONLY GROUP } \\
\hline & $\begin{array}{c}\text { Experimental } \\
\quad(n=64)\end{array}$ & $\begin{array}{l}\text { Control } \\
(n=57)\end{array}$ & $\begin{array}{l}\text { Post-Test-Only } \\
\quad(n=43)\end{array}$ \\
\hline Hours of crying & $1.29(1.21)$ & $2.94(3.17)$ & $5.91(4.28)$ \\
\hline Intensity of crying & $1.97(1.32)$ & $2.79(1.83)$ & $4.37(1.06)$ \\
\hline
\end{tabular}

nificant difference in treatment and control group responses. Fifty-eight percent of the mothers in the control group were satisfied with their care and $95 \%$ of the mothers in the treatment group reported they were satisfied with their care $(\mathrm{p}<0.001)$.

Resolution of crying was also assessed by using the exit interview data and these showed significant differences in the two randomized groups, $x^{2}(1)=4.27, p<0.04$. Eighty-three percent of the parents in the treatment group reported that their infant's irritability was totally resolved at the exit interview. Sixty-eight percent of the control group parents reported resolution. Only 4\% of the infants in the treatment group were unresolved compared to $18.5 \%$ of the infants in the control group in which the unexplained irritability still persisted at the 8-week follow-up visit. Average infant age at time of resolution of irritability was $9.2 \pm 2.7$ weeks with no statistically significant difference in the two randomized groups (Figure 4).

\section{DISCUSSION}

An increased understanding of both the infant's and parent's contribution to the puzzling phenomenon of unexplained infant irritability has been gained through a series of studies conducted by these investigators. An intervention strategy, the REST Routine for intervening with irritable infants and their families was developed from a proposed theoretical perspective and from the findings of previously conducted studies. The needs of the parents and the understanding gained regarding the maternal experience was incorporated into the intervention model. The basic concepts of synchrony, entrainment, and support formed the conceptual framework for the intervention program.

Thus the findings from this clinical trial support the current view of infant colic or unexplained irritability as a developmental, behavioral pattern that resolves over time and is responsive to environmental modification and structured, cue-based care. The results indicate a consis- 


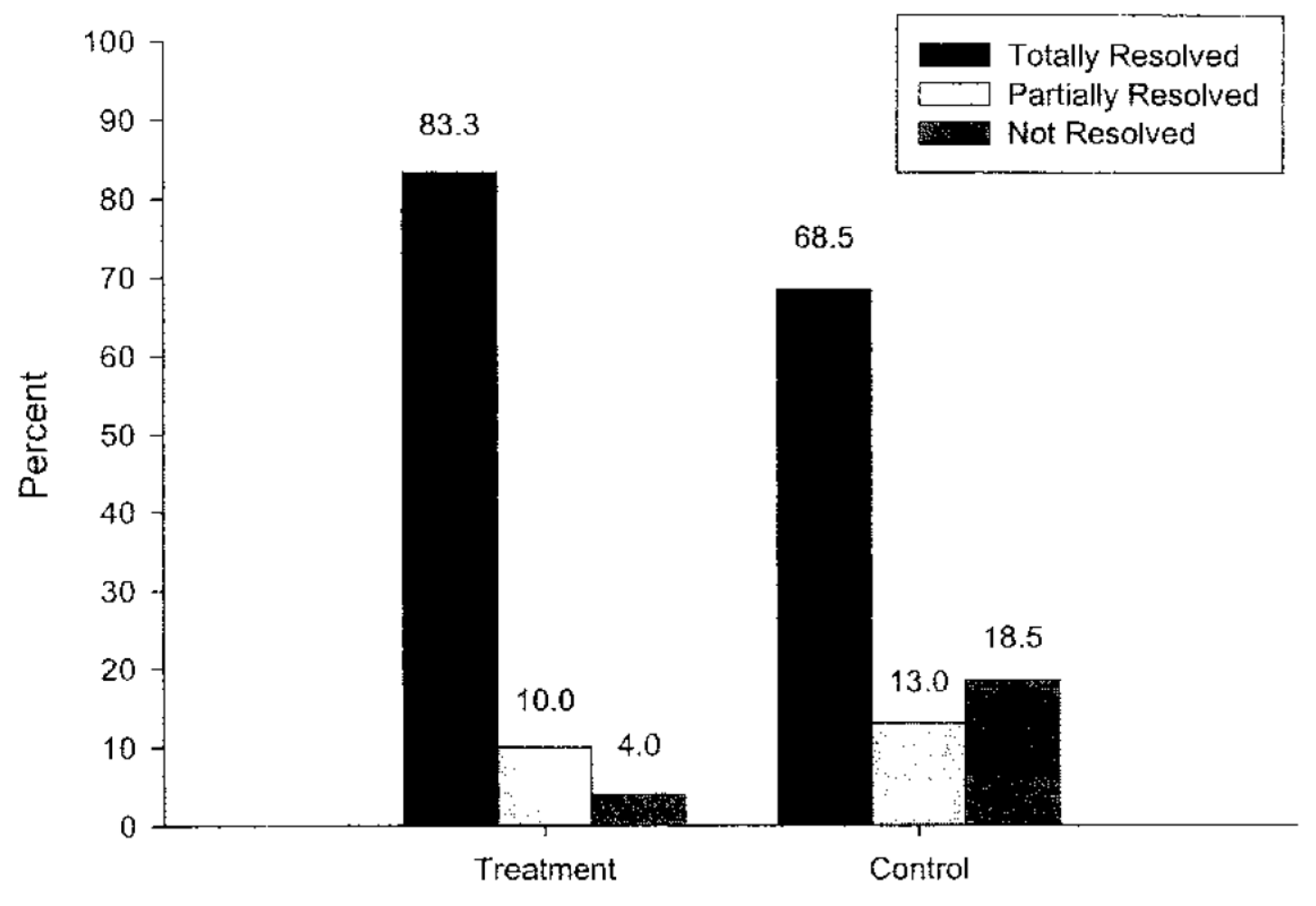

Study Group

tent pattern of reduction in irritability over time as a consistent feature of this developmental state regulation disorder. In all cases, the level of irritability decreased across the eight time points of measurement. However, in addition, the pattern of decline in the treatment group was sharper than the decline for infants in the control group. This apparent difference in trajectories was statistically significant as indicated by the time by treatment interactions. This interaction effect supports the hypothesis that the intervention reduced the amount of crying and fussiness in the treatment group.

Several conclusions and observations can be drawn from this study. Families in both treatment and control groups reported benefiting from a nurse visiting in their home to inquire about their infant and their well-being. A third, post-test-only, group of infants was enrolled to study the effects of the nurse data collection visits. These infants received only one visit at a point in time that was comparable to the postintervention visits in the other groups. These parents reported significantly higher levels of infant crying and distress. Participants in this study were representative of a subset of families with irritable infants who were referred due to persistent, unexplained crying in their infants or entered this study through self-referral by persistent and motivated parents. In general these families were resourceful and well educated. The cost-benefit ratio of this 4-week, home- based intervention program and replication of these findings in other populations of families with irritable infants warrants further investigation. Options for targeting the program for those most in need, and evaluation of other delivery modes for the intervention, are also important areas for further research. 
The REST Routine's potential for reducing excess infant irritability and providing relief from the distress seen in these families has been demonstrated in this systematic, randomized clinical trial. This intervention program and the theoretically based approach to working with these families can be very useful to practitioners working with families. The treatment program developed emphasizes the individualized needs and unique features of each family. Irritable infants with unclear cues and chaotic home environments responded well to protection from overstimulation and overload. Daily routines that reinforced regularity and predictability in sleeping and feeding were critical components of the management strategy. Information and validation of parental competency was stressed and opportunities to express feelings to an empathetic, supportive professional were key components of this home-based intervention for irritable infants.

\section{REFERENCES}

1. Barr RG, Hopkins B, Green JA, eds. Crying as a Sign, a Symptom, \& a Signal: Clinical Emotional and Developmental Aspects of Infant and Toddler Crying. New York: Cambridge University Press; 2000.

2. Barr RG, Rotman A, Yaremko J, et al. The crying of infants with colic: a controlled empirical description. Pediatrics. 1992;90(1 Pt 1):14-21.

3. Helseth S, Begnum S. A comprehensive definition of infant colic: parents' and nurses' perspectives. J Clin Nurs. 2002;11:672-680.

4. Barr RG, St. James-Roberts I, Keefe MR. New Evidence on Unexplained Early Infant Crying: Its Origins, Nature and Management. New Brunswick, NJ: Johnson \& Johnson Pediatric Roundtable; 2001.

5. St. James-Roberts I. Infant crying levels, and maternal patterns of care, in normal community and clinically referred samples. In: Lester B, ed. Biological and Social Aspects of Infant Crying. New York: Plenum Press; 2000.

6. Carbaugh SF. Understanding shaken baby syndrome. Adv Neonatal Care. 2004;4:105-116.

7. Castiglia PT. Shaken baby syndrome. J Pediatr Health Care. 2001;15:78-80.

8. Papousek M. Persistent infant crying, parenting and infant mental health. In: Osofsky J, Fitzgerald H, eds. WAIMH Handbook of Infant Mental Health. Vol. 4. New York: John Wiley \& Sons; 2000.

9. Papousek M, von Hofacker N. Persistent crying in early infancy: a non-trivial condition of risk for the developing mother-infant relationship. Child Care Health Dev. 1998;24:395-424.

10. Levitzky S, Cooper R. Infant colic syndrome-maternal fantasies of aggression and infanticide. Clin Pediatrics. 2000;39:395-400.

11. Wood RM, Gustafson GE. Infant crying and adults' anticipated caregiving responses: acoustic and contextual influences. Child Dev. 2001;72:1287- 1300.

12. Raiha H, Lehtonen L, Korhonen T, Korvenranta H. Family functioning 3 years after infantile colic. J Dev Behav Pediatr. 1997;18:290-294.

13. Stifter CA, Bono MA. The effect of infant colic on maternal self-perceptions and motherinfant attachment. Child Care Health Dev. 1998;24:339-351.

14. Stifter CA, Spinrad TL. The effect of excessive crying on the development of emotion regulation. Infancy. 2002;3:133-152.

15. Morris S, James-Roberts IS, Sleep J, Gillham P. Economic evaluation of strategies for managing crying and sleeping problems. Arch Dis Child. 2001;84:15-19. 
16. Pinyerd BJ. Strategies for consoling the infant with colic: fact or fiction? J Pediatr Nurs. 1992; 7:403-411.

17. Keefe MR, Froese-Fretz A. Living with an irritable infant: maternal perspectives. MCN Am J Matern Child Nurs. 1991;16:255-259.

18. Swadling C, Griffiths P. Is modified cow's milk formula effective in reducing the symptoms of infant colic? Br J Commun Nurs. 2003;8:24-27.

19. Estep D, Kulczycki A Jr. Treatment of infant colic with amino acid-based infant formula: a preliminary study. Acta Paediatr. 2000;89:22-27.

20. Hill DJ, Hosking CS. Infantile colic and food hypersensitivity. J Pediatr Gastroenterol Nutr. 2000;30:67-76.

21. Keefe MR. Irritable infant syndrome: theoretical perspectives and practice implications. Adv Nurs Sci. 1988;10:70-78.

22. Hiscock H, Wake M. Randomised controlled trial of behavioural infant sleep intervention to improve infant sleep and maternal mood. BMJ. 2002;324:1062-1065.

23. Garrison MM, Christakis DA. A systematic review of treatments for infact colic. Pediatrics. 2000;106:184-190.

24. St James-Roberts I, Sleep J, Morris S, et al. Use of a behavioural programme in the first 3 months to prevent infant crying and sleeping problems. J Paediatr Child Health. 2001;37:289-297.

25. Keefe MR, Barbosa GA, Froese-Fretz A, et al. An intervention program for families with irritable infants. MCN Am J Matern Child Nurs. 2005;30:230- 236.

26. Keefe M, Kotzer AM, Froese-Fretz A, Curtin M. A longitudinal comparison of irritable and nonirritable infants. Nurs Res. 1996;45:4-9.

27. Keefe MR, Froese-Fretz A, Kotzer AM. Newborn predictors of infant irritability. J Obstet Gynecol Neonatal Nurs. 1998;27:513-520.

28. Emde RN, Gaensbauer TJ, Harmon RJ. Emotional expression in infancy; a biobehavioral study. Psychol Issues. 1976;10:1-200.

29. Diggle P, Liang K, Zeger S. Analysis of Longitudinal Data. New York: Oxford University Press; 2000.

30. Stokes M, Davis C, Kioch G. Categorical Data Analysis Using SAS System. 2nd ed. Cary, NC: SAS Institute; 2000. 